\title{
SALUD MENTAL EN NIÑOS Y ADOLESCENTES DE LA RIOJA: RESULTADOS DE LA ENCUESTA NACIONAL DE SALUD DE ESPAÑA 2011/12 (ENSE 2011/12)
}

\author{
Natalia Zaldívar Allona \\ Alicia Pérez de Albéniz Iturriaga \\ Universidad de La Rioja \\ Eduardo Fonseca Pedrero \\ Universidad de La Rioja \\ Centro de Investigación Biomédica en Red de Salud Mental
}

RESUMEN: Los trastornos mentales son un problema de salud global, no obstante, en La Rioja, se han Ilevado a cabo pocos estudios empíricos que traten de analizar los problemas emocionales y comportamentales en población infanto-juvenil. El objetivo principal fue conocer el estado de salud mental en los niños y adolescentes riojanos de 4 a 14 años, así como su relación con distintos indicadores de salud física y hábitos de vida saludables. La muestra la conformaron un total de 101 participantes $(M=9,44 ; D T=3,12 ; 57$ hombres; 44 mujeres), extraídos de la Encuesta Nacional de Salud de España 2011/12. Se utilizaron el Strengths and Difficulties Questionnaire versión heteroinforme y el KIDSCREEN-10, para evaluar la salud mental y la calidad de vida relacionada con la salud, respectivamente. También se emplearon preguntas sobre hábitos de vida saludable (comida, actividad física, sueño) y trastornos mentales previos diagnosticados. Los resultados indicaron que el $5 \%$ de la muestra riojana presentó riesgo de mala salud mental y el $8,9 \%$ obtuvo puntuaciones límites. La subescala problemas emocionales del SDQ se asoció de forma negativa con las puntuaciones del KIDSCREEN-10. Los participantes considerados "de riesgo" no refirieron una peor calidad de vida ni una mayor prevalencia de trastornos mentales en comparación con el grupo de "no riesgo". No se encontró relación entre riesgo de mala salud mental y los hábitos de alimentación (a excepción del consumo de comida rápida), la actividad física o el descanso. Estos hallazgos permiten tener una visión actual sobre el estado de salud mental de los jóvenes riojanos de cara a destinar recursos e implementar programas de promoción del bienestar emocional.

PALABRAS CLAVE: Salud mental, prevalencia, La Rioja, hábitos de vida, calidad de vida. 


\title{
MENTAL HEALTH IN CHILDREN AND ADOLESCENTS IN LA RIOJA: RESULTS FROM THE NATIONAL HEALTH SURVEY IN SPAIN (ENSE 2011/12)
}

\begin{abstract}
Mental disorders are a global health problem, however, in La Rioja, few empirical studies have been carried out that try to analyze emotional and behavioral problems in The child and adolescent population. The main goal was to analyze the mental health state of Riojan Youth from 4 to 14 years old, as well as their relationship with physical health and healthy lifestyle habits. The sample was 101 participants ( $M=9.44, S D=3.12,57$ men, 44 women). It was extracted from the National Health Survey of Spain 2011/12. The Strengths and Difficulties Questionnaire parent's version and KIDSCREEN-10 were used to evaluate mental health and quality of life, respectively. Questions about healthy habits (food, physical activity, sleep) and previous mental disorders were also used. The results indicated that $5 \%$ of the sample presented higher scores (at risk for mental health problems), and $8.9 \%$ obtained borderline scores. The SDQ emotional problems subscale was negatively associated with the KIDSCREEN-10 scores. Participants considered "at risk" did not report a worse quality of life or a higher prevalence of previous mental disorders compared to the "no risk" group. No relationship was found between the higher risk of mental health problems and eating habits (except for the consumption of fast food), physical activity or rest. These findings allow us to have a current vision on the state of mental health of Riojan youth in order to allocate resources and implementing programs to promotion emotional wellbeing.
\end{abstract}

KEYWORDS: Mental health, prevalence, La Rioja, life habits, quality of life.

Recibido: 06/09/2017

Aceptado: 09/01/2018

Correspondencia: Eduardo Fonseca Pedrero, Universidad de La Rioja, C/ Luis de Ulloa, s/n, 26004 Logroño. Email: eduardo.fonseca@unirioja.es.

La Organización Mundial de la Salud (OMS) estima que una de cada cuatro personas padecerá algún tipo de trastorno mental a lo largo de su vida (OMS, 2005). Los trastornos mentales representan un $12,5 \%$ de todos los problemas médicos, siendo superior al cáncer y a los trastornos cardiovasculares (FEAFES, 2008). Además, entre el $15-20 \%$ de los niños y adolescentes sufren de un problema mental, y cerca de la mitad de ellos se manifiestan antes de los 15 años manteniéndose estable hasta la edad adulta (Davies et al., 2015; Kessler et al., 2007; Polanczyk, Salum, Sugaya, Caye, y Rohde, 2015). En la Encuesta Nacional de Salud España 2006 (ENSE) (Bones Rocha, Pérez, Rodríguez-Sanz, Borrell, y Obiols, 2010; Ministerio de Sanidad y Consumo Español, 2006), se encontró que entre el 19,2 y el 26,6\% de los niños y adolescentes presentaban riesgo de sufrir mala salud mental, siendo grave 
en el 4-6\% de los casos. Concretamente, en La Rioja, un estudio reciente realizado con el Cuestionario de Capacidades y Dificultades (Strengths and Difficulties Questionnaire, SDQ) (Goodman, 1997), en una muestra representativa de adolescentes, mostró que el 7,7\% manifestaron posible riesgo de mala salud mental $(9,4 \%$ de las mujeres; 5,8\% de los hombres) (Fonseca-Pedrero, 2017).

Dentro de las consecuencias más importantes que se derivan de los trastornos de salud mental se encuentran las económicas, sociales, sanitarias, familiares, educativas y personales (Gore et al., 2011; OMS, 2006). El impacto económico de los trastornos mentales es difícil de medir, ya que solo se pueden cuantificar algunos de los costos asociados. Según el III Plan Regional de Salud Mental de La Rioja 2016-2020, en la Unión Europea el coste social y económico que suponen los trastornos mentales corresponde aproximadamente al $4 \%$ del Producto Nacional Bruto, unos 182.000 millones de euros. El presupuesto para salud mental de la mayoría de los países es inferior al 1\% del gasto total en salud (OMS, 2004). Según un estudio de Parés-Badell et al. (2014), en España en 2010 se dedicaron 46.000 millones de euros a problemas relacionados con la salud mental. Además, según Lara et al. (2014), en el año 2010 en España, los trastornos neuropsiquiátricos supusieron el $18,4 \%$ del total de años de vida ajustados por discapacidad (AVAD) y la cuarta causa de muerte en 2012. En el ámbito educativo, los problemas de salud mental también repercuten negativamente en el rendimiento académico y en la tasa del fracaso escolar (Fonseca-Pedrero, 2017; Matalí, 2016; Schulte-Koerne, 2016).

La salud mental no solo se refiere a la ausencia de enfermedad sino a un estado de bienestar físico, mental y social. La calidad de vida relacionada con la salud (CVRS), se refiere a la evaluación subjetiva de la influencia del estado de salud, los cuidados sanitarios y la promoción de la salud sobre la capacidad del individuo para mantener un nivel de funcionamiento que le permite realizar las actividades que le son importantes, y que afectan a su estado general de bienestar (Shumaker y Naughton, 1995). Con frecuencia la CVRS se considera un indicador de bienestar subjetivo o de bienestar emocional. Por ejemplo, la ENSE 2011-2012 (Ministerio de Sanidad, Servicios Sociales e Igualdad, 2013) midió la CVRS de la población entre 8 y 14 años a través del test KIDSCREEN-10 (Aymerich et al., 2005). Los resultados fueron satisfactorios, ya que mostraron una puntuación media de 87,9 puntos sobre 100, nivel comparable con el de los países europeos con mejores indicadores de salud infantil. Asimismo, Fonseca-Pedrero (2017) encontró que un 71,9\% de adolescentes riojanos refirieron niveles adecuados de bienestar emocional subjetivo (puntuación igual o superior a 7 en una escala de 10 puntos).

La salud mental y la CVRS están muy relacionada con los hábitos de vida saludable. En La Rioja existen datos algo alarmantes sobre hábitos que perjudican tanto la salud física como mental de la población infanto-juvenil. A los 11 años un alto porcentaje de niños comienza a fumar; el 31\% de la población infantil tiene sobrepeso, de ellos el $12 \%$ es obeso. Además, La Rioja es la comunidad autónoma donde antes se inicia el consumo de bebidas alcohólicas (13 años) y el 23,78\% de la población infantil no hace ningún ejercicio al aire libre (Ministerio de Sanidad, Servicios Sociales e Igualdad, 2013). Los alimentos que se ingieren afectan más allá de la salud física, Ilegando a modificar también las emociones, sentimientos y com- 
portamientos de las personas. Un estudio realizado por Oddy et al. (2009) afirmó que los adolescentes con peor salud mental presentaban un aumento en el consumo de comida rápida, carne roja precocinada y productos de bollería y repostería. En cambio, comer cinco piezas de frutas y verduras diariamente reduce el riesgo de desarrollar enfermedades no transmisibles y ayuda a garantizar una ingesta suficiente de fibra dietética. Por su parte, un estudio británico indicó que un patrón alimentario rico en "comida basura" a la edad de cuatro años se asociaba con el aumento de la hiperactividad, problemas de conducta y problemas entre iguales a los siete años (Wiles, Northstone y Lewis, 2009).

Otro de los hábitos saludables más importantes para mantener una buena salud general es la realización de actividad física. Son numerosas las investigaciones que defienden los beneficios de la actividad física en diferentes ámbitos del bienestar psicológico, ya que, entro otros aspectos, mejora la salud subjetiva, el estado de ánimo, reduce la depresión, disminuye los niveles de ansiedad, favorece la regulación del estrés e incrementa la autoestima (Biddle y Asare, 2011; Janssen y Leblanc, 2010). En cuanto al tipo de ejercicio que realizar, estudios como el de Edwards et al., (2004) señalan que tanto la actividad física aeróbica como el entrenamiento en resistencia contribuyen a mejorar el bienestar psicológico de las personas, defendiendo la idea de que más que el tipo de ejercicio a practicar, lo importante es el tiempo dedicado. Algunos autores como Jiménez et al., (2008) indican que por debajo de los 90 minutos semanales no se consiguen los beneficios deseados y perseguidos sobre la salud percibida, el estrés y los estados afectivos como la tristeza o la fatiga, por lo que afirman que la asignatura de educación física en las escuelas tiene un papel muy relevante. Con respecto al descanso, numerosos estudios se han centrado en los efectos que tiene el sueño en nuestro organismo. Por ejemplo, Astil et al. (2012) destacó en una de sus revisiones que el sueño insuficiente se asocia con un déficit en las funciones cognitivas de orden superior (analizar, sintetizar) y con un aumento en los problemas de comportamiento en los niños. Esta hipótesis es defendida por expertos que afirman que dormir entre siete y nueve horas diarias ayuda a mantener un correcto rendimiento físico e intelectual.

Hasta el momento en La Rioja se han Ilevado a cabo pocos estudios empíricos que traten de analizar y comprender la expresión de los problemas emocionales y comportamentales en una muestra representativa, extraída de forma aleatoria de la población general, a partir de la ENSE 2011/12. Además, escasos trabajos han analizado la relación entre las diferentes dificultades emocionales y comportamentales, la calidad de vida y el estilo de vida saludable. El objetivo general de esta investigación fue conocer el estado de salud mental en los niños y adolescentes de 4 a 14 años de La Rioja, así como su relación con distintos indicadores de salud física y hábitos de vida saludables. Dentro de este contexto de investigación se plantean los siguientes objetivos específicos en relación a una muestra representativa de alumnos de La Rioja: a) analizar la prevalencia de riesgo de mala salud mental en población infanto-juvenil riojana; b) analizar la relación existente entre el estado de salud mental y la CVRS; c) explorar la relación entre el estado de salud mental y los buenos hábitos de alimentación; d) examinar la relación entre el estado de salud mental y la actividad física y el descanso; y e) analizar la asociación entre el estado de salud mental y trastornos mentales previos diagnosticados. 


\section{Método}

\section{Participantes}

Los participantes de este estudio forman parte de una muestra representativa de la población analizada en la ENSE 2011-2012 (Ministerio de Sanidad, Servicios Sociales e Igualdad, 2013). Este muestreo fue trietápico estratificado, donde las unidades de primera etapa son las secciones censales y las de segunda etapa son las viviendas principales. De cada hogar se eligió a un adulto de 15 años o más para cumplimentar el Cuestionario de Adultos y en caso de que hubiera menores se seleccionó uno aleatoriamente para que fuera cumplimentado el Cuestionario de Menores. Este último fue resuelto por la madre, el padre o el tutor en el 94\% de los menores. Los cuestionarios y metodología completa se pueden consultar en la página web: http://www. msssi.gob.es/estadisticas/microdatos.do.

Los datos que se recogen en la ENSE 2011-2012 corresponden a N=5495 entrevistas a menores, de las cuales se eliminaron $n=1667$ por no haber utilizado con ellos el SDQ y $n=3727$ por no pertenecer a la Comunidad Autónoma de La Rioja. La muestra final utilizada fue de 101 participantes, 57 hombres $(56,4 \%)$ y 44 mujeres $(43,6 \%)$. La media de edad fue de 9,44 años $(D T=3,25)$, oscilando el rango de edad entre 4 y 14 años. Además, cabe destacar que el 93,1\% de los participantes eran de origen español, mientras que el 6,9\% eran de otra nacionalidad.

\section{Instrumentos}

Cuestionario de Capacidades y Dificultades versión heteroinforme (Strengths and Difficulties Questionnaire, SDQ) (Goodman, 1997)

EI SDQ es un instrumento de medida utilizado para la evaluación de dificultades conductuales y emocionales, así como la valoración de capacidades en el ámbito social (Ortuño-Sierra, Fonseca-Pedrero, Inchausti y Sastre i Riba, 2016). También se ha utilizado como una herramienta de cribado psicopatológico. Se compone de 25 ítems con un formato de respuesta Likert de tres opciones 0 (No, nunca), 1 ( $A$ veces), 2 (Sí, siempre). Los ítems se agrupan en cinco subescalas (con cinco ítems cada una): Dificultades Emocionales, Problemas de Conducta, Dificultades de Hiperactividad, Problemas con Compañeros y Conducta Prosocial. Las primeras cuatro subescalas forman una puntuación Total de Dificultades. A mayor puntuación mayor nivel de dificultades emocionales y comportamentales, excepto para la subescala de Conducta Prosocial, donde una menor puntuación se corresponde con un peor ajuste en el comportamiento. EI SDQ ha mostrado adecuadas propiedades psicométricas en trabajos previos tanto nacionales como internacionales (Mason, Chmelka y Thompson, 2012; Ortuño-Sierra et al., 2015, Ortuño-Sierra, Aritio-Solana y Fonseca-Pedrero, 2017).

\section{Cuestionario KIDSCREEN-10 versión para padres (Aymerich et al., 2005)}

Es el primer instrumento de evaluación de CVRS para la población infantil y adolescente, desarrollado simultáneamente en trece países europeos (Berra et al., 2009). Esta versión del KIDSCREEN-10 está diseñada para la población de 8 a 14 años. Consta de 10 preguntas de las cuales se elimina una de la versión original 
autoadministrada (¿Tus padres te han tratado de forma justa?) y la sustituye una pregunta de salud general percibida con un formato de respuesta tipo Likert de cinco opciones (muy bueno, bueno, regular, malo, muy malo). Las nueve preguntas originales presentan un período recordatorio de una semana y se contestan con el mismo formato yendo desde 0 (Nada o Nunca) hasta 5 (Siempre o Muchísimo). Las respuestas deben ser proporcionadas por un informante indirecto que será el padre, madre, tutor o persona bien informada para ello, pero proporcionando la perspectiva del niño (Ministerio de Sanidad, Servicios Sociales e Igualdad, 2013). El rango de puntuaciones es de 0-100, representando una mejor CVRS cuanta más puntuación. EI KIDSCREEN-10 ha mostrado adecuadas propiedades psicométricas en trabajos previos (Parizi et al., 2014; Urzúa et al., 2009).

\section{Evaluación de hábitos alimenticios saludables}

Dentro de la ENSE 2011-2012 para medir los hábitos alimenticios saludables de los participantes se les realizó tres preguntas:

1. El hábito de no desayunar antes de salir de casa se consideró a partir de la pregunta “¿Dónde desayuna habitualmente?". Esta es respondida mediante 1(En casa, antes de salir), 2 (Fuera de casa) o 3 (No suele desayunar).

2. La frecuencia de consumo de comida rápida se consideró a partir de la pregunta "¿Con qué frecuencia consume los siguientes alimentos?: pollo frito, hamburguesas, pizzas, bocadillos." Las respuestas eran tipo Likert de 7 opciones (1= "A diario", 2= "Tres o más veces a la semana, pero no a diario", $3=$ "Una o dos veces a la semana", 4= "Menos de una vez a la semana", 5= "Nunca o casi nunca", 8= "No sabe", 9= "No contesta"). Se considera la respuesta positiva cuando responden "tres o más veces a la semana" o "a diario".

3. La frecuencia de consumo de verduras se consideró a partir de la pregunta "¿Con qué frecuencia consume los siguientes alimentos?: Verduras, ensaladas y hortalizas". A esta pregunta se respondió de igual manera que la anterior, con un formato tipo Likert de 7 opciones. Se considera una frecuencia positiva cuando se responde "tres o más veces a la semana" o "a diario".

\section{Evaluación del ejercicio físico y sueño}

Para analizar la actividad física que realizan los niños y adolescentes se contestó a la pregunta “¿Cuál de estas posibilidades describe mejor la frecuencia con la que realiza alguna actividad física en su tiempo libre?" siendo la contestación tipo Likert con 6 opciones ( 1 = "No hace ejercicio", 2 = "Hace alguna actividad física o deportiva ocasional", 3= "Hace actividad física varias veces al mes", 4= "Hace entrenamiento deportivo o físico varias veces a la semana", $8=$ "No sabe", 9= "No contesta"). Se considera físicamente activo cuando se responde con el número 4.

Con respecto al descanso, se les realizó la pregunta “¿Cuántas horas duerme habitualmente al día?" en el que se debían de incluir también las horas de siesta. Se consideró un descanso positivo en aquellos casos en los que la respuesta se situó entre 7 y 9 horas. 


\section{Evaluación del diagnóstico de los trastornos mentales}

Para analizar si existe una asociación entre la salud mental de los alumnos y los trastornos mentales respondieron a la pregunta " $i$ Ha padecido alguna vez trastornos de la conducta (incluye hiperactividad)?", "¿Ha padecido alguna vez trastornos mentales (depresión, ansiedad...)?". Dichas preguntas son respondidas mediante (1= "Sí", 6= "No", 8= "No sabe" 9= "No contesta"). Se considera positivo si se responde con un 1 .

\section{Procedimiento}

La presente investigación forma parte de la ENSE 2011-2012 realizada por el Ministerio de Sanidad, Servicios Sociales e Igualdad en colaboración con el Instituto Nacional de Estadística (INE).

El contacto inicial con los hogares se realizó a través de una carta del INE solicitando su colaboración, en la cual se les informaba a los participantes que habían sido seleccionados para realizar la encuesta, el carácter confidencial de la misma y el aviso de la próxima visita que realizaría un entrevistador acreditado. Los responsables encargados de la recogida de datos recibieron unos cursos de formación con una duración de 3 días en horario de mañana y tarde, mientras que las personas a su cargo que debían estar en cada zona determinada realizaron un curso cuya duración fue de 2 días también en horario de mañana y tarde.

Las entrevistas se realizaron en dos fases, en la primera se administró el Cuestionario de Hogar y en la segunda el Cuestionario de Adultos (15 años o más) y el Cuestionario de Menores (de 0 a 14 años). Para esta investigación se precisa únicamente este último. La información se obtuvo indirectamente a través del padre, madre o tutor, pudiendo ser contestada por otra persona autorizada y bien informada en caso de incapacidad de los mismos. El método de recogida de información se realizó a través de una entrevista personal asistida por ordenador, pudiendo ser una entrevista telefónica en casos excepcionales. Los trabajos de campo (recogida de datos, inspección, seguimiento y control de la información correspondiente a cada provincia) se Ilevaron a cabo por la empresa Investigación, Planificación y Desarrollo IPD, adjudicataria del contrato publicitado por el INE, bajo la supervisión de este. Se puede encontrar esta información y otras relacionadas con la metodología a seguir durante toda la investigación en: https://www.msssi.gob.es/estadEstudios/estadisticas/encuestaNacional/encuestaNac2011/MetodologiaENSE2011_12.pdf.

\section{Análisis de datos}

Con la finalidad de investigar los objetivos propuestos se llevaron a cabo los siguientes análisis de datos.

En primer lugar, para examinar la prevalencia de las dificultades emocionales y comportamentales se calcularon los porcentajes de participantes en cada una de las tres franjas de puntuaciones del SDQ (No caso, Límite y Probable caso). Aunque es una muestra extraída de forma aleatoria de la población y representativa, el número de participantes riojanos fue reducido, por lo que también se decidió construir, en base a trabajos previos, dos franjas de puntuaciones (No caso y Límite y Probable caso de mala salud mental). 
En segundo lugar, con la finalidad de examinar la relación existente entre el estado de salud mental y la calidad de vida se computó la matriz de correlaciones de Pearson entre las puntuaciones de los instrumentos de medida. Asimismo, se realizó una prueba $t$ para muestras independientes considerando los dos grupos de participantes con factor fijo y la CVRS como variable dependiente.

En tercer lugar, con la finalidad de explorar la relación entre los dos grupos de salud mental y los hábitos de alimentación, la práctica de ejercicio físico y el descanso y los trastornos mentales diagnosticados se analizaron las tablas de contingencia. Se utilizó la prueba de chi-cuadrado para comprobar si existían diferencias estadísticamente significativas $(p<0,05)$.

Los análisis de datos se llevaron a cabo con el programa estadístico SPSS v22 (IBM, 2013).

\section{Resultados}

\section{Estado de salud mental en niños y adolescentes de La Rioja}

Considerando la puntuación Total de Dificultades del SDQ se analizó la prevalencia del estado de salud mental utilizando dos (No caso, Probable caso) y tres franjas (No caso, Límite, Probable caso) de puntuaciones. En primer lugar, de los 101 participantes en el estudio el $86,1 \%$ de la muestra no mostró riesgo de sufrir un problema de salud mental, el 8,9\% puntuaron límite y el 5\% se situó en el grupo de riesgo de mala salud mental. En segundo lugar, en la Tabla 1 se muestra el porcentaje de participantes en función de los puntos de corte del SDQ en dos grupos (No caso vs Probable caso) tanto para las subescalas como para la puntuación Total de Dificultades del SDQ.

Tabla 1. Prevalencia (\%) de No caso y Probable caso de riesgo de mala salud mental en La Rioja en función de las puntuaciones del Strengths and Difficulties Questionnaire (SDQ)

\begin{tabular}{lcc}
\hline SDQ & No caso & Probable caso \\
\hline Dificultades emocionales & 88,1 & 11,9 \\
Problemas de conducta & 76,2 & 23,8 \\
Problemas con compañeros & 89,1 & 10,9 \\
Dificultades de Hiperactividad & 72,3 & 27,7 \\
Conducta prosocial & 97,0 & 3,0 \\
Total Dificultades & 86,1 & 13,9 \\
\hline
\end{tabular}

Si se compara con el conjunto de España, y como se puede ver en la Figura 1, hubo un mayor porcentaje de No casos en La Rioja frente al conjunto total de España $(82,8 \%)$. Sin embargo, las puntuaciones Límite en La Rioja fueron superiores, siendo en España de un 8,5\%. Por último, los Probables casos de mala salud mental en España fueron el 8,7\% frente al 5\% de La Rioja. Además, en la Figura 2 se presenta la comparativa con el resto de CCAA de los Probables casos de mala salud mental. 


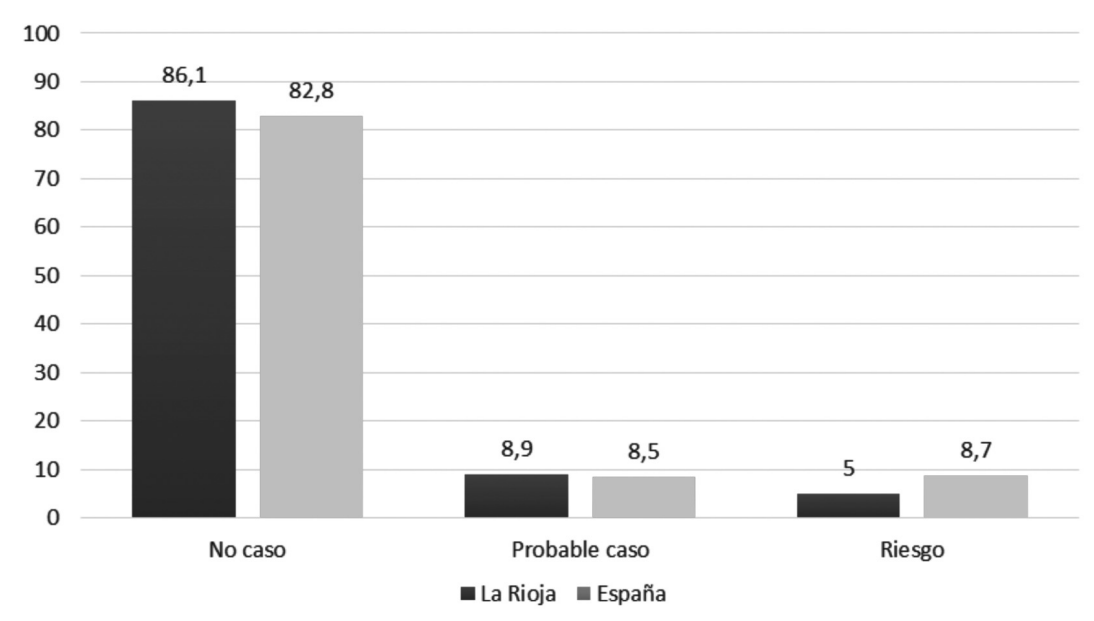

Figura 1. Prevalencia de los tres grupos de salud mental en La Rioja y en España según los puntos de corte del Strengths and Difficulties Questionnaire

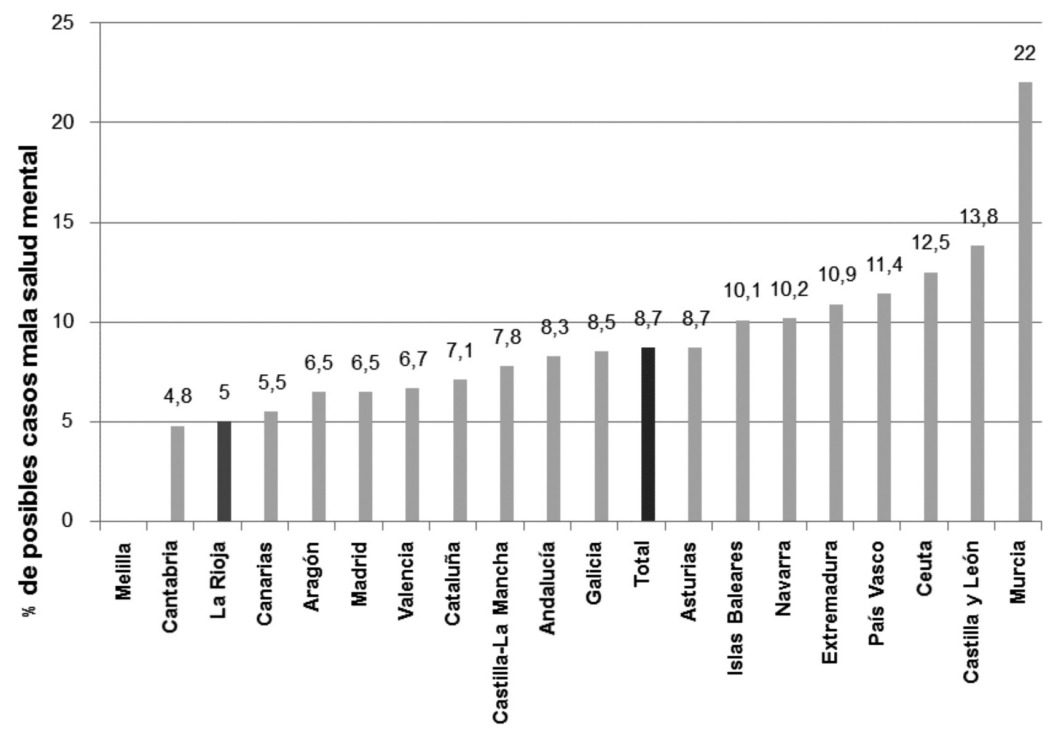

Figura 2. Comparación entre Comunidades Autónomas

en la prevalencia de riesgo de mala salud mental según los puntos de corte del Strengths and Difficulties Questionnaire

\section{Relación entre el estado de salud mental y la calidad de vida relacionada con la salud}

A continuación, se examinó la relación entre el estado de salud mental y la CVRS. Antes de comenzar se analizó la distribución de las puntuaciones en la muestra de 
participantes riojanos. Los resultados se recogen en la Figura 3. Seguidamente se computaron las correlaciones de Pearson entre las puntaciones del SDQ y del KIDSCREEN-10. Como se puede observar en la Tabla 2 únicamente se encontró una asociación estadísticamente significativa entre la subescala Problemas Emocionales del SDQ y la puntuación del KIDSCREEN-10.

Dentro de este apartado también se realizó una comparación de medias para examinar si los participantes considerados "Probable caso" de mala salud mental presentaban una peor CVRS con respecto al grupo de "No caso". Los resultados de la prueba $t$ para muestras independientes mostraron que no se encontraron diferencias estadísticamente significativas entre los dos grupos $\left(t_{(66)}=0,713 ; p=0,479\right)$. En la Figura 4 se presentan las puntuaciones medias en calidad de vida para los dos grupos de salud mental.

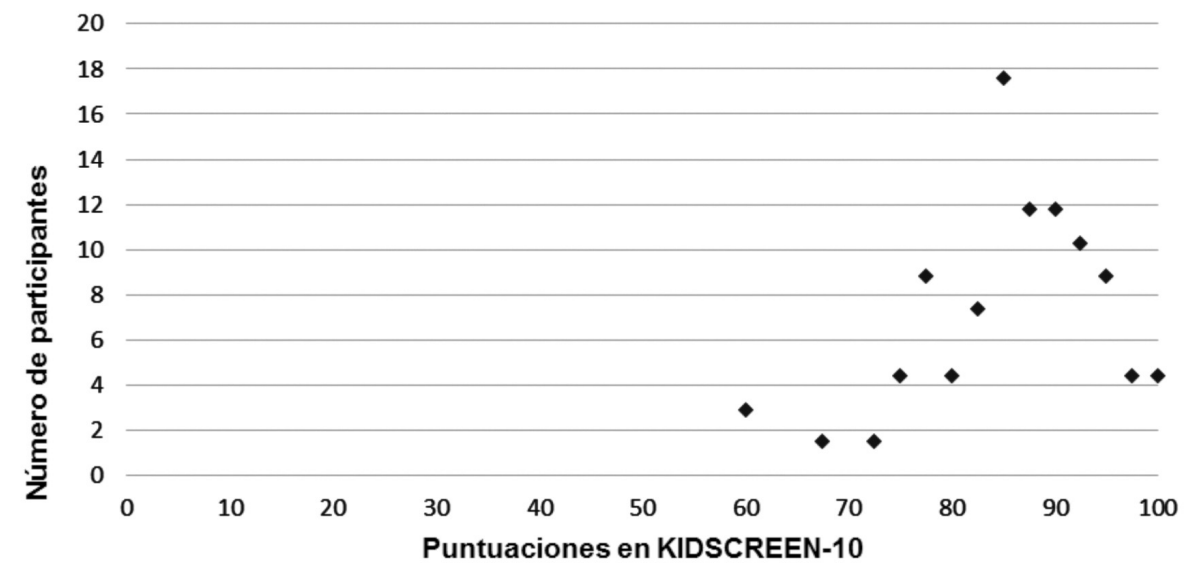

Figura 3. Distribución de puntuaciones en calidad de vida relacionada con la salud para los participantes riojanos

Tabla 2. Matriz de correlaciones de Pearson entre las puntuaciones en salud mental y calidad de vida en los participantes de La Rioja

\begin{tabular}{lccccccc}
\hline & 1 & 2 & 3 & 4 & 5 & 6 & 7 \\
\hline KIDSCREEN-10 (1) & 1 & & & & & & \\
Dificultades Emocionales (2) & $-0,29^{*}$ & 1 & & & & & \\
Problemas de conducta (3) & 0,05 & $0,25^{*}$ & 1 & & & & \\
Problemas con compañeros (4) & $-0,06$ & $0,26^{* *}$ & 0,14 & 1 & & & \\
Dificultades de Hiperactividad (5) & $-0,07$ & 0,13 & $0,51^{* *}$ & 0,02 & 1 & & \\
Conducta Prosocial (6) & 0,02 & $-0,02$ & $-0,29^{* *}$ & 0,06 & $-0,14$ & 1 & \\
Total Dificultades SDQ (7) & $-0,14$ & $0,56^{* *}$ & $0,74^{* *}$ & $0,42^{* *}$ & $0,79^{* *}$ & $-0,17$ & 1 \\
\hline
\end{tabular}

Nota. ${ }^{* *} p<0,01 ;{ }^{*} p<0,05$. SDQ: Strengths and Difficulties Questionnaire 


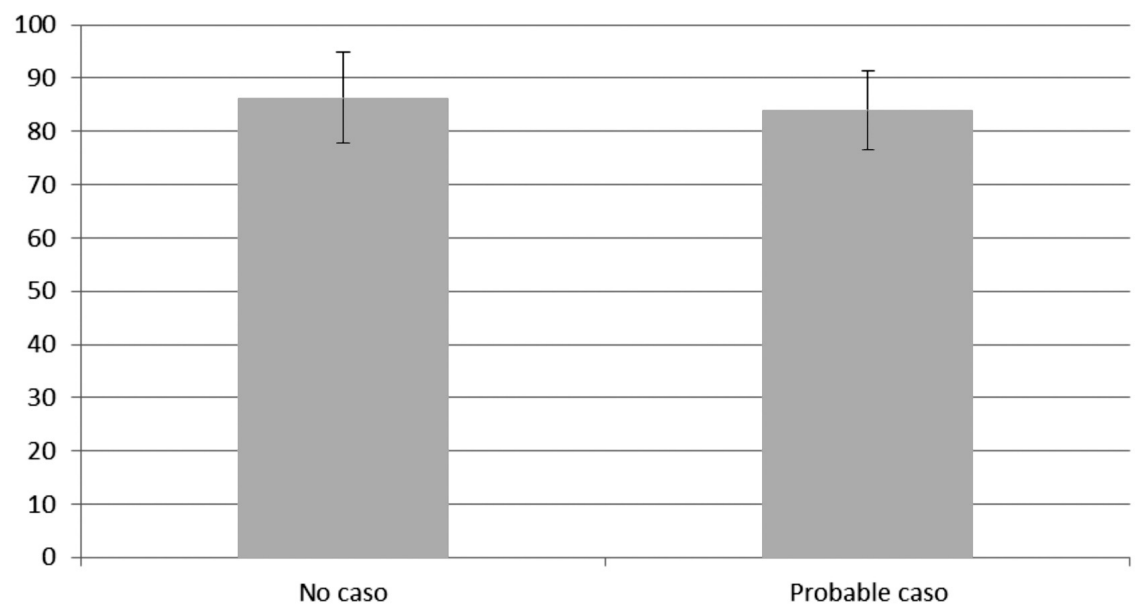

Figura 4. Puntuaciones medias y desviaciones típicas en calidad de vida relacionada con la salud en los grupos de salud mental en función de las puntuaciones en el Strengths and Difficulties Questionnaire

\section{Análisis del estado de salud mental con respecto a la importancia de buenos hábitos de alimentación}

Con respecto a los hábitos alimentarios más comunes entre los participantes riojanos, se analizó las respuestas sobre la frecuencia de consumo de verduras y comida rápida, cuyos resultados aparecen en la Figura 5.

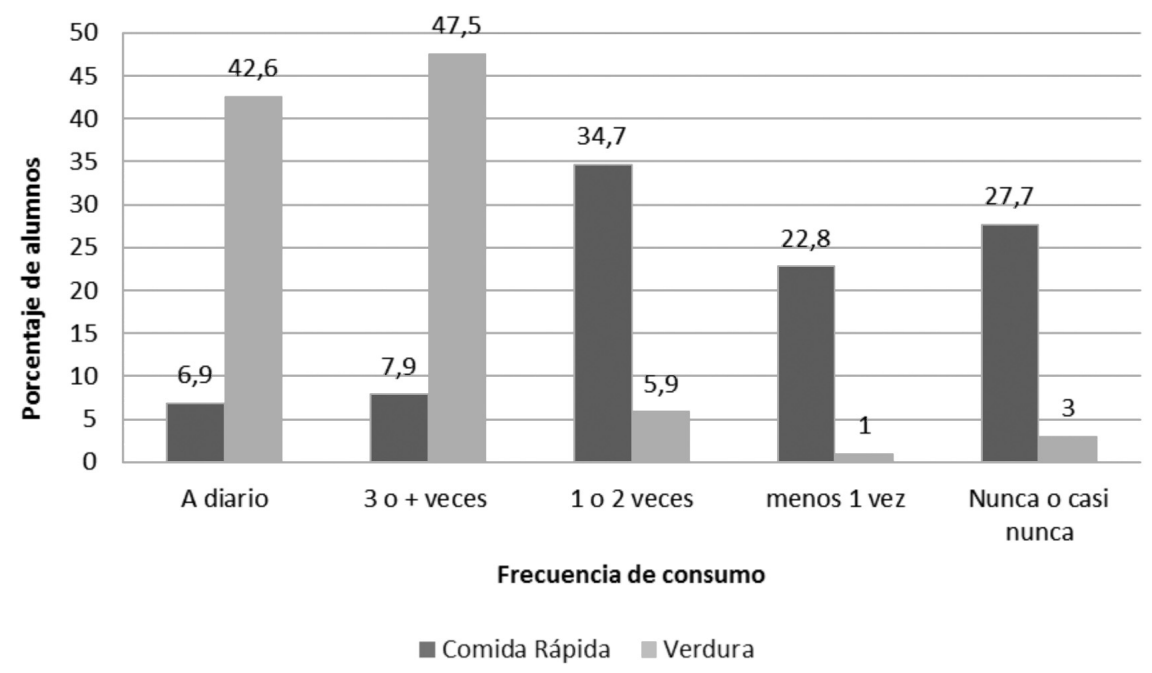

Figura 5. Frecuencia en el consumo de verduras y comida rápida en los niños y jóvenes riojanos 
A continuación, se analizó la relación entre los grupos de salud mental y los hábitos alimentarios. Según las tablas de contingencia, no se encontraron diferencias estadísticamente significativas cuando se comparó la frecuencia de consumo de verduras entre los dos grupos de No caso y Probable caso de mala salud mental $\left(\chi_{(1)}^{2}=1,786 ; p=0,181\right)$.

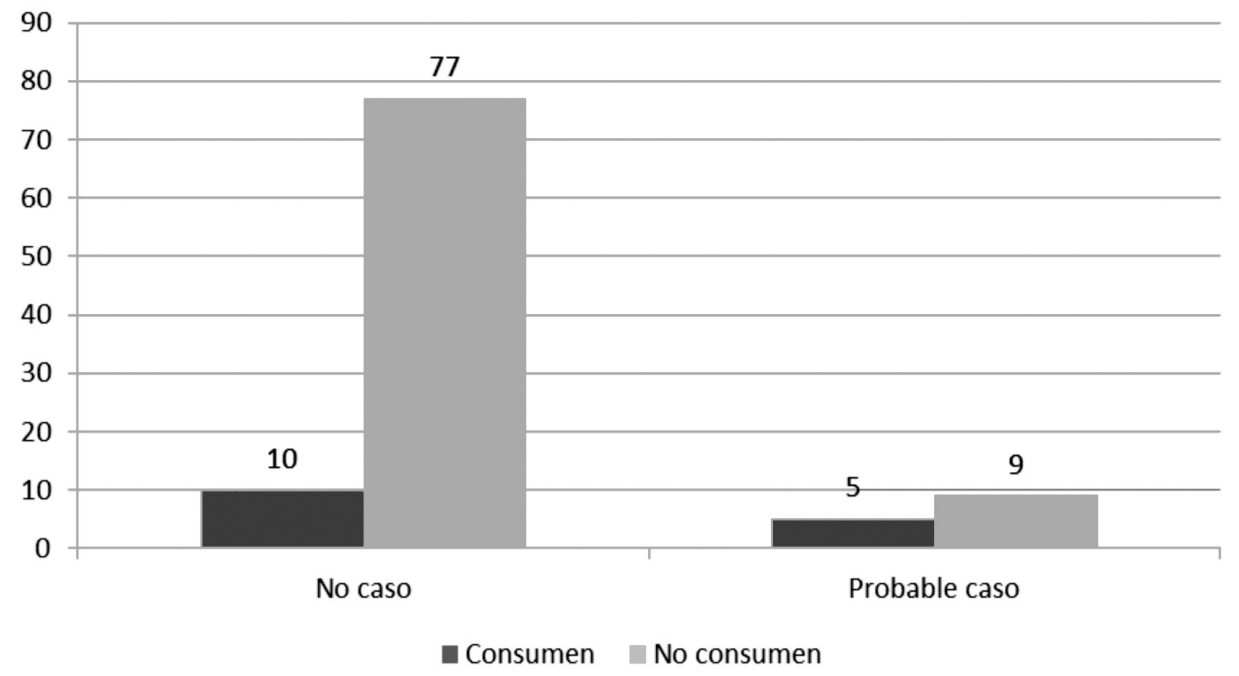

Figura 6. Consumo de comida rápida en ambos grupos en función de los dos grupos de salud mental

Por otro lado, al comparar la frecuencia de consumo de comida rápida entre los dos grupos de salud mental, sí se encontraron diferencias estadísticamente significativas $\left(\chi^{2}{ }_{(1)}=5,594 ; p=0,018\right)$, cuyos resultados aparecen en la Figura 6 . Resaltar que más de la mitad de los alumnos considerados como Probables Casos de mala salud mental consumían comida rápida a diario o como mínimo tres veces a la semana.

\section{Relación entre el nivel de salud mental y la actividad física en la población infanto-juvenil riojana}

Otro de los hábitos de vida saludable que se analizó fue la realización de actividad física. En primer lugar se analizó el patrón de actividad física en España y en La Rioja (Figura 7). A continuación, se analizó la relación entre los grupos de salud mental y la actividad física. Según las tablas de contingencia, no se encontraron diferencias estadísticamente significativas cuando se comparó la frecuencia de realización de actividad entre los dos grupos de No caso y Probable caso de mala salud mental $\left(\chi_{(1)}^{2}=0,265 ; p=0,606\right)$. Los resultados se muestran en la Figura 8. 


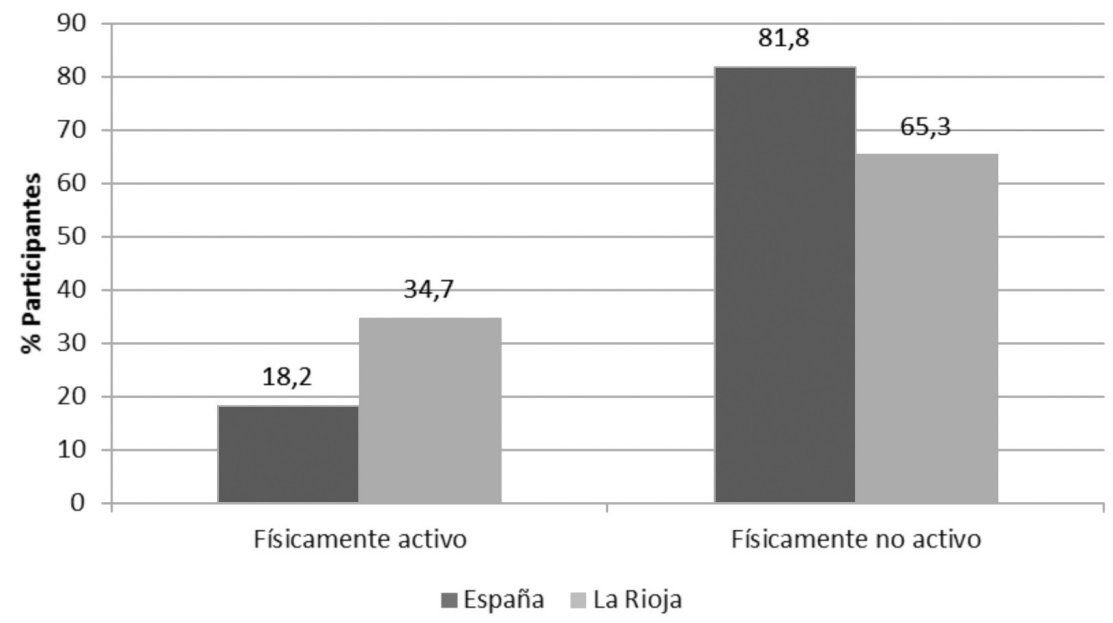

Figura 7. Frecuencia de los participantes físicamente activos de La Rioja frente al conjunto nacional

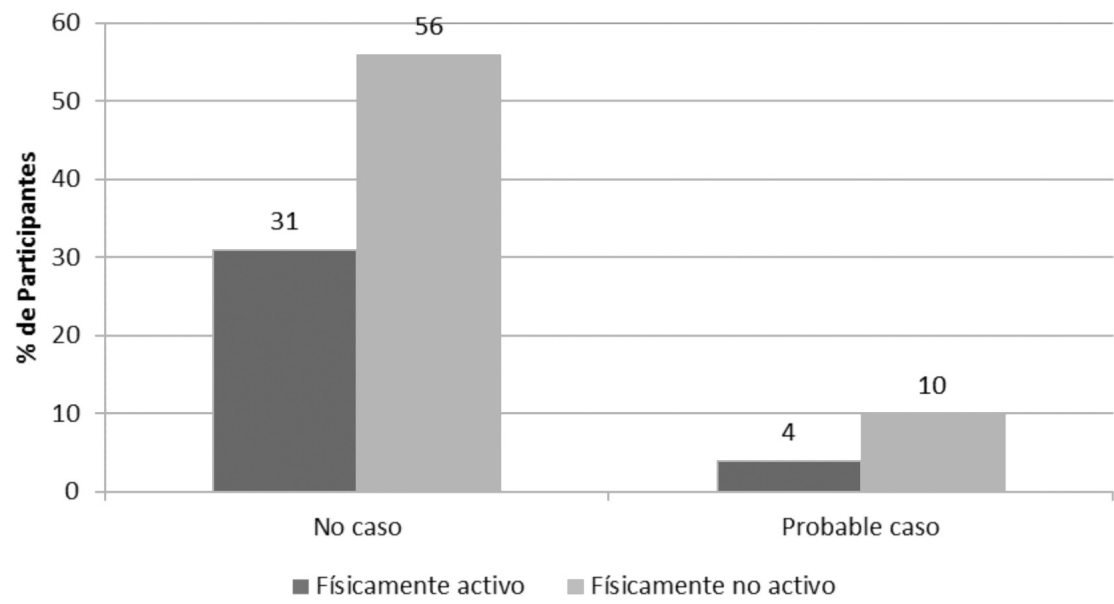

Figura 8. Frecuencia de actividad física en los dos grupos de salud mental en función del Strengths and Difficulties Questionnaire

\section{Análisis de la asociación entre el estado de salud mental y trastornos mentales en alumnos de La Rioja}

Por último, se analizó la relación existente entre la salud mental de los alumnos riojanos y sus trastornos mentales. De los $N=101$ participantes en el estudio, dos de ellos afirmaron tener un diagnóstico médico que confirmara tener al menos un trastorno mental. Además, un participante presentó un diagnóstico médico que confirmó un trastorno de la conducta. Es digno de mención que ninguno de los tres 
participantes respondió positivamente a ambas preguntas. Tras realizar tablas de contingencia entre la presencia de un trastorno mental y los dos grupos de salud mental, no se observaron diferencias estadísticamente significativas $\left(\chi^{2}{ }_{(1)}=2,232\right.$; $p=0,135)$. Por el contrario, si se encontraron diferencias estadísticamente significativas entre los trastornos de conducta y los dos grupos de salud mental $\left(\chi^{2}{ }_{(1)}=\right.$ $6,276 ; p=0,012)$. En la Figura 9 se pueden observar los resultados de estas tablas de contingencia.

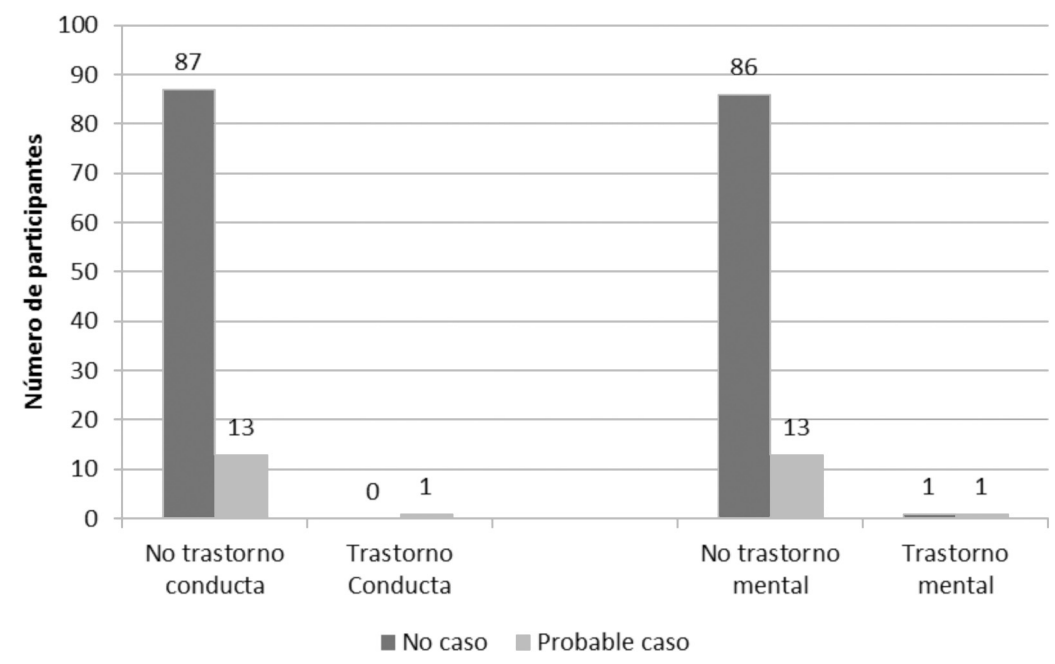

Figura 9. Relación entre los trastornos de conducta y los trastornos mentales con los dos grupos de salud mental en función del Strengths and Difficulties Questionnaire

\section{Discusıón}

Hasta el momento se han llevado a cabo pocos trabajos rigurosos que traten de analizar y comprender el estado de salud mental en una muestra representativa de la población riojana infanto-juvenil. El principal propósito de esta investigación fue conocer el estado de salud mental, a través del SDQ, en los niños y adolescentes de 4 a 14 años de La Rioja, así como su relación con la CVRS, distintos indicadores de hábitos de vida saludables (comida, actividad física, sueño) y trastornos mentales previos diagnosticados.

En primer lugar, se analizó la prevalencia de riesgo de mala salud mental en la población infanto-juvenil riojana según las puntuaciones del SDQ. Los resultados mostraron que el $5 \%$ de la muestra total presentó riesgo de mala salud mental, y el $8,9 \%$ obtuvo puntuaciones límites. Comparándola con la muestra total española, La Rioja es la tercera Comunidad Autónoma con menor porcentaje de probables casos de mala salud mental. Estos resultados van en consonancia con los obtenidos en estudios como el de Ortuño-Sierra et al. (2014), que tras administrar el SDQ a 508 estudiantes de La Rioja y Asturias, concluyeron que el 9\% de los participantes 
habían obtenido puntuaciones límites en el SDQ- versión autoinforme, mientras que el $6 \%$ presentaban riesgo de mala salud mental. Por su parte, en el estudio de Barriuso-Lapresa et al. (2014) se obtuvieron puntuaciones más elevadas utilizando la versión para padres del SDQ, declarando que los problemas de salud mental afectan entre un 10 y un $20 \%$ de los niños y adolescentes. Finalmente, en un estudio realizado por Fonseca-Pedrero (2017), se observó que el 78,9\% de los adolescentes riojanos presentaron, según las puntuaciones del SDQ, una adecuada salud mental, el $13,4 \%$ se halló en la franja de posible riesgo de salud mental y el 7,7\% manifestó riesgo de mala salud mental. En términos generales, los resultados encontrados en este estudio son similares a los hallados en la literatura previa (Bones et al., 2010; Davies et al., 2015; Fonseca Pedrero y Debbané, 2017; Kessler et al., 2007; Polanczyk et al., 2015) y ponen de manifiesto, por un lado, que un conjunto de la población infanto-juvenil presenta riesgo de mala salud mental, y por otro, que un grupo más amplio de los jóvenes refiere alguna dificultad en el ajuste emocional y/o comportamental.

En segundo lugar, se analizó la relación existente entre el estado de salud mental y la CVRS en La Rioja. Los resultados en las puntuaciones del KIDSCREEN-10 estuvieron en torno a los 85 puntos, valor que indica una adecuada CVRS en los niños y jóvenes riojanos pero ligeramente inferior a la obtenida en la media nacional. Además, únicamente se encontró una asociación estadísticamente significativa entre la subescala problemas emocionales del SDQ y la puntuación del KIDSCREEN-10. Este resultado posiblemente sea debido al reducido tamaño muestral empleado. Trabajos previos han Ilegado a resultados similares. Por ejemplo, Fonseca-Pedrero (2017), encontró una correlación de $r=-0,45(p<0,01)$ entre la puntuación total de Dificultades del SDQ y el ítem 1 (satisfacción general con la vida) del Índice de Bienestar Personal-versión escolar (PWI-SC) (Cummins y Lau, 2005). Por último, se observó que las puntuaciones medias en CVRS del grupo considerado como "No caso" eran ligeramente superiores a los del grupo "Probable caso", si bien no se encontraron diferencias estadísticamente significativas. Estos resultados van en consonancia con el estudio de Ravens-Sieberer et al. (2013), el cual afirmó que los alumnos con algún tipo de problema en su salud física o mental puntúan más bajo que los niños sanos. Otro estudio de Ravens-Sieberer et al. (2008), que relaciona el SDQ con el KIDSCREEN, también mostró que cuanto mayor es la puntuación en el SDQ, peor calidad de vida es referida por el niño. A modo de resumen, los resultados hallados en este estudio son similares a los encontraros en la literatura previa, con lo que se puede afirmar que existe una asociación negativa entre problemas emocionales y comportamentales y CVRS.

En tercer lugar, se examinó la relación entre el estado de salud mental de los niños y adolescentes y sus hábitos de alimentación. Se analizó la frecuencia con la que los riojanos consumen verduras y comida rápida. Casi la mitad de los participantes afirmaron comer verdura tres veces o más a la semana, pero hay que destacar que un $3 \%$ de los niños y adolescentes no comen nunca o casi nunca este tipo de alimentos. Con respecto a la comida rápida, algo más de una cuarta parte de los participantes no la consume nunca o casi nunca, dato que se considera como un buen indicador de hábitos alimenticios. Por el contrario, es preocupante que el 6,9\% lo consuman a diario. Al comparar estos hábitos con los dos grupos de salud mental establecidos por 
el SDQ (No caso, Probable caso) se puede afirmar que aquellos alumnos considerados como "Probable caso" consumen más comida rápida que los "No caso". Estos resultados van en consonancia con los obtenidos en el estudio Kulkarni et al. (2015) que defendieron la idea de que las dietas altas en alimentos poco saludables se asocian con una mayor probabilidad de síntomas de mala salud mental. Por su parte, un estudio de Blanchflower et al. (2013) indicó que existía una asociación positiva entre la salud mental y el consumo de frutas y hortalizas. Aunque algunos de los estudios previos realizados no estén en consonancia con los resultados encontrados en esta investigación, generalmente se puede afirmar que los hábitos alimenticios están relacionados con la salud física y mental.

En cuarto lugar, se estudió la relación entre el nivel de salud mental en la población infanto-juvenil riojana y sus hábitos de actividad física. El 34,7\% de los participantes riojanos afirmaron realizar actividad física varias veces a la semana superando el porcentaje de la población total española. A continuación, se analizó la relación entre los grupos de salud mental establecidos en el SDQ (No caso y Probable caso) y la actividad física, no encontrando diferencias estadísticamente significativas. Nuevamente, los resultados encontrados posiblemente se deban al escaso tamaño muestral. En contraposición a estos resultados, un estudio de Almagro et al. (2014) afirmó que existe una asociación inversa entre actividad física y la probabilidad de sufrir una enfermedad mental como la depresión, incluso cuando la actividad física es de baja intensidad. En la misma línea, el estudio de Jiménez et al. (2008) concluyó que las personas que realizan regularmente ejercicio físico se perciben más saludables, con menor estrés y tienen mejor estado de ánimo que aquellas que no realizan ejercicio. Sin embargo, este mismo autor afirma que el efecto sobre otros trastornos mentales como la ansiedad no está demostrado. En general, y aunque no hay gran consenso en la literatura previa sobre de qué manera el ejercicio físico afecta a la salud mental, lo cierto es que en estos estudios se manifiesta que realizar actividad física sí afecta en nuestro organismo.

En quinto y último lugar, se analizó la asociación existente entre el estado de salud mental y los trastornos mentales previos diagnosticados clínicamente. Los resultados mostraron que dos participantes manifestaron tener al menos un trastorno mental y uno un trastorno de la conducta. En total, se puede decir que un 3\% de los participantes tenía mala salud mental. Además, tras realizar las tablas de contingencia entre los dos grupos de salud mental establecidos por el SDQ (No caso y Probable caso) y los trastornos mentales de los participantes, se observa que el alumno con trastorno de la conducta se sitúa en el grupo "Probable caso". Por el contrario, en el caso de los dos alumnos con trastornos mentales, uno de ellos pertenece al grupo "Probable caso" y el otro al grupo "No caso". Los resultados encontrados pueden ser debidos a múltiples factores, como por ejemplo, que el SDQ fue cumplimentado por el padre, madre o tutor del alumno, el escaso tamaño muestral, o a las limitaciones de propio instrumento de medida referidas a su capacidad discriminativa y predictiva.

Existen ciertas limitaciones en este estudio y a los resultados obtenidos. En primer lugar, la muestra riojana que se utilizó proviene del estudio ENSE 2011-2012 y era limitada. Al contar con solo 101 participantes, algunos análisis no contaban con la suficiente potencia estadística para arrojar diferencias estadísticamente significativas. A pesar de ello, la manera aleatoria de escoger a los participantes es una fortaleza 
de la investigación, ya que fueron seleccionados a través de un excelente muestreo. En segundo lugar, con respecto a los instrumentos utilizados, cabe destacar que al ser los padres o tutores los responsables de contestar por los niños, los resultados son totalmente subjetivos y se pueden encontrar sesgos y limitaciones similares a las reportadas por los autoinformes. En tercer lugar, en algunas variables existen preguntas que no han sido respondidas, reduciendo la muestra de análisis aún más. En cuarto lugar, es un trabajo de naturaleza transversal por lo que no se pueden hacer inferencias de naturaleza causal.

A la luz de los hallazgos del presente trabajo sería interesante realizar el mismo estudio con una muestra más representativa de la población infanto-juvenil riojana. Además, y dado el impacto social, económico y personal asociado a los problemas psicológicos, se deberían proponer medidas para la promoción de la salud mental y el bienestar emocional, por ejemplo, implementando programas de educación emocional, tanto para la población general como para la población infanto-juvenil. Los jóvenes son el principal capital de nuestra sociedad y la mejor inversión de futuro.

\section{AgradeCimientos}

Esta investigación ha sido financiada por el Ministerio de Ciencia e Innovación de España (MICINN) (referencia PSI 2014-56114-P), por el Instituto Carlos III, Centro de Investigación Biomédica en Red de Salud Mental (CIBERSAM), por la convocatoria 2015 de "Ayudas Fundación BBVA a Investigadores y Creadores Culturales" y por las "Ayudas Fundación BBVA a Equipos de Investigación Científica 2017".

\section{ReFERENCIAS BIBLIOGRÁFICAS}

Almagro, S., Dueñas, M. A. y Tercedor, P. (2014). Actividad física y depresión: revisión sistemática. Revista Internacional de Medicina y Ciencias de la Actividad Física y el Deporte, 14(54), 377-392.

American Psychiatric Association (2013). Diagnostic and statistical manual of mental disorders (5th ed.). Washington, DC: Author.

Astill, R. G., Van der Heijden, K. B., Van Ijzendoorn, M. H. y Van Someren, E. J. W. (2012). Sleep, cognition, and behavioral problems in school-age children: A century of research meta-analyzed. Psychological Bulletin, 138(6), 1109-1138.

Aymerich, M., Berra, S., Guillamón, I., Herdman, M., Alonso, J., Ravens-Sieberer, U. y Rajmil, L. (2005). Desarrollo de la versión en español del KIDSCREEN, un cuestionario de calidad de vida para la población infantil y adolescente. Gaceta Sanitaria, 19(2), 93-102.

Barriuso-Lapresa, L. M., Hernando-Arizaleta, L. y Rajmil, L. (2014). Reference values of the Strengths and Difficulties Questionnaire (SDQ) version for parents in the Spanish population, 2006. Actas Españolas de Psiquiatría, 42, 43-48.

Berra, S., Bustingorry, V., Henze, C., Rajmil, L. y Butinof, M. (2009). Adaptación transcultural del cuestionario KIDSCREEN para medir calidad de vida relacionada con la salud en población argentina de 8 a 18 años. Archivos Argentinos de Pediatria, 107(4), 307-314. 
Biddle, S. J. H. y Asare, M. (2011). Physical activity and mental health in children and adolescents: A review of reviews. British Journal of Sports Medicine, 45(11), 886-95.

Blanchflower, D.G., Oswald, A. J. y Stewart-Brown, S. (2013). Is Psychological Well-Being Linked to the Consumption of Fruit and Vegetables? Social Indicators Research, 114(3), 785-801.

Bones, K., Pérez, K., Rodríguez-Sanz, M., Borrell, C. y Obiols, J. E. (2010). Prevalencia de problemas de salud mental y su asociación con variables socioeconómicas de trabajo y salud: resultados de la Encuesta Nacional de Salud de España. Psicothema, 22, 389-395.

Cummins, R. A. y Lau, A. D. L. (2005). Personal Wellbeing Index-School Children (PWI-SC) (3rd Ed.). Melbourne: Deakin University.

Davies, S. J., Pearson, R. M., Stapinski, L., Bould, H., Christmas, D. M., Button, K. S., ... Evans, J. (2015). Symptoms of generalized anxiety disorder but not panic disorder at age 15 years increase the risk of depression at 18 years in the Avon Longitudinal Study of Parents and Children (ALSPAC) cohort study. Psychological Medicine, 46(1), 73-85.

Edwards, D. J., Edwards, S. D. y Basson, C. J. (2004). Psychological well-being and physical self-esteem in sport and exercise. International Journal of Mental Health Promotion, 6, 25-32.

FEAFES-Confederación Española de Agrupaciones de Familiares y Personas con Enfermedad Mental (2008). Salud mental y medios de comunicación. Guía de estilo.

Fonseca-Pedrero, E. (Ed.) (2017). Bienestar emocional en adolescentes riojanos. Logroño: Universidad de La Rioja.

Fonseca Pedrero, E. y Debbané, M. (2017). Schizotypal traits and psychotic-like experiences during adolescence: An update. Psicothema, 29, 5-17.

Gallagher, S. K. y Mechanic, D. (1996). Living with the mentally ill: effects on the health and functioning of other members. Social Science and Medicine, 42, 1691-1701.

Goodman, R. (1997). The Strengths and Difficulties Questionnaire: A research note. Journal Child Psychology and Psychiatry, 38(5), 581-586.

Gore, F. M., Bloem, P. J., Patton, G. C., Ferguson, J., Joseph, V., Coffey, C., Sawyer S. M. y Mathers, C. D. (2011). Global burden of disease in young people aged 10-24 years: a systematic analysis. Lancet, 377, 2093-2102.

Janssen, I. y Leblanc, A. G. (2010). Systematic review of the health benefits of physical activity and fitness in school-aged children and youth. The International Journal of Behavioral Nutrition and Physical Activity, 7, 40.

Jiménez, M. G., Martínez, P., Miró, E. y Sánchez, A. I. (2008). Bienestar psicológico y hábitos saludables: ¿están asociados a la práctica de ejercicio físico? International Journal of Clinical and Health Psychology, 8(1), 185-202.

Kessler, R. C., Amminger, G. P., Aguilar-Gaxiola, S., Alonso, J., Lee, S. y Ustün, T. B. (2007). Age of onset of mental disorders: a review of recent literature. Current Opinion in Psychiatry, 20(4), 359-364.

Kulkarni, A. A., Swinburn, B. A. y Utter, J. (2015). Associations between diet quality and mental health in socially disadvantaged New Zealand adolescents. European Journal of Clinical Nutrition, 69(1), 79-83. 
Lara, E., Garín, N., Ferrari, A. J., Tyrovolas, S., Olaya, B., Sánchez, L., ... Haro, J. M. (2014). The Spanish burden of disease 2010: Neurological, mental and substance use disorders. Revista de Psiquiatría y Salud Mental, 8(4), 207-217.

Leiva, L., George, M., Simonsohn, A., Antivilo, A., Squicciarini, A. M., Vargas, B. y Guzman, J. (2015). Salud mental escolar: Logros de una intervención preventiva en salud mental en niños y niñas del primer ciclo de enseñanza básica. Psicoperspectivas, 14, 31-41.

Lluch Canut, M. T. (1999). Construcción de una escala para evaluar la salud mental positiva. (Tesis doctoral no publicada). Universidad de Barcelona.

Mason, W. A., Chmelka, M. B. y Thompson, R. W. (2012). Responsiveness of the Strengths and Difficulties Questionnaire (SDQ) in a sample of high-risk youth in residential treatment. Child and Youth Care Forum, 41(5), 479-492.

Matalí, J. L. (2016). Adolescentes con trastornos de comportamiento. ¿Cómo podemos detectarlos? ¿Qué se debe hacer? Hospital San Joan de Déu, Barcelona.

Ministerio de Sanidad, Servicios Sociales e Igualdad. (2013). Encuesta Nacional de Salud 2011-2012. Instituto Nacional de Estadística, 1-12. Recuperado de https:// www.msssi.gob.es/estadEstudios/estadisticas/encuestaNacional/encuesta2011.htm.

Ministerio de Sanidad y Consumo Español (2012). Encuesta Nacional de Salud 20062007. Madrid: Autor.

Oddy, W. H., Robinson, M., Ambrosini, G. L., OSullivan, T. A., de Klerk, N. H., Beilin, L. J., ... Stanley, F. J. (2009). The association between dietary patterns and mental health in early adolescence. Preventive Medicine, 49(1), 39-44.

Organización Mundial de la Salud (2001). Salud mental: nuevos conocimientos, nuevas esperanzas. Informe sobre la salud en el mundo, 169. Ginebra: OMS. Recuperado de http://www.who.int/whr/2001/es/

Organización Mundial de la Salud (2004). Invertir en Salud mental. Ginebra: OMS. Recuperado de http://www.who.int/mental_health/advocacy/en/spanish_final.pdf.

Organización Mundial de la Salud (2005). Prevención de los Trastornos Mentales: Intervenciones Efectivas y Opciones Políticas. Ginebra: OMS. Recuperado de http://www.who.int/mental_health/evidence/Prevention_of_mental_disorders_ spanish_version.pdf.

Organización Mundial de la Salud. (2006). Manual de recursos de la OMS sobre Salud Mental, Derechos humanos y legislación, Ginebra: OMS. Recuperado de http://www.who.int/mental_health/policy/legislation/WHO_Resource_Book_ MH_LEG_Spanish.pdf.

Ortuño-Sierra, J., Aritio-Solana, R. y Fonseca-Pedrero, E. (2017). Mental health difficulties in children and adolescents: The study of the SDQ in the Spanish National Health Survey 2011-2012. Psychiatry Research, 259, 236-242.

Ortuño-Sierra, J., Fonseca-Pedrero, E., Inchausti, F. y Sastre i Riba, S. (2016). Evaluación de dificultades emocionales y comportamentales en población infanto-juvenil: El cuestionario de capacidades y dificultades (SDQ). Papeles del Psicólogo, 37(1), 14-26.

Ortuño-Sierra, J., Fonseca-Pedrero, E., Aritio-Solana, R., Velasco, A. M., de Luis, E. C., Schumann, G., ... Lawrence, C. (2015). New evidence of factor structure and measurement invariance of the SDQ across five European nations. European Child and Adolescent Psychiatry, 24(12), 1523-1534. 
Ortuño-Sierra, J., Fonseca-Pedrero, E., Paino, M. y Aritio-Solana, R. (2014). Prevalencia de síntomas emocionales y comportamentales en adolescentes españoles. Revista de Psiquiatría y Salud Mental, 7(3), 121-130.

Parés-Badell, O., Barbaglia, G., Jerinic, P., Gustavsson, A., Salvador-Carulla, L. y Alonso, J. (2014). Cost of disorders of the brain in Spain. PLoS ONE, 9(8).

Parizi, A. S., Garmaroudi, G., Fazel, M., Omidvari, S., Azin, S. A., Montazeri, A. y Jafarpour, S. (2014). Psychometric properties of KIDSCREEN health-related quality of life questionnaire in Iranian adolescents. Quality of Life Research, 23(7), 21332138. http://doi.org/10.1007/s11136-014-0655-2.

Paula-Pérez, I. y Martos-Pérez, J. (2009). Síndrome de asperger y autismo de alto funcionamiento: Comorbilidad con trastornos de ansiedad y del estado de ánimo. Revista de Neurología, 48, Suppl 2, S31-34.

Polanczyk, G. V., Salum, G. A., Sugaya, L. S., Caye, A. y Rohde, L. A. (2015). Annual research review: A meta-analysis of the worldwide prevalence of mental disorders in children and adolescents. Journal of Child Psychology and Psychiatry and Allied Disciplines, 56, 345-365.

Ravens-Sieberer, U., Herdman, M., Devine, J., Otto, C., Bullinger, M., Rose, M., y Klasen, F. (2013). The European KIDSCREEN approach to measure quality of life and well-being in children: development, current application, and future advances. Quality of Life Research, 23(3), 791-803.

Ravens-Sieberer, U., Erhart, M., Gosch, A. y Wille, N. (2008). Mental health of children and adolescents in 12 European countries.Results from the European KIDSCREEN study. Clinical Psychology \& Psychotherapy, 15(3), 154-163.

Rodríguez, F. (2009). Aspectos explicativos de comorbilidad en los TGD, el síndrome de Asperger y el TDAH: Estado de la cuestión. Revista Chilena de Neuropsicología, 4(1), 12-19.

Shumaker, S. y Naughton, M. (1995). The International Assessment of Health-Related Quality of Life: a theoretical perspective. En Shumaker, S. y Berson, R. (Eds.), The international assessment of healthrelated quality of life: theory, translation, measurement and analysis. Oxford: Rapid Communications.

Schulte-Koerne, G. (2016). Mental health problems in a school setting in children and adolescents. Deutsches Arzteblatt International, 113(11), 183.

Urzúa, A., Cortés, E., Vega, S., Prieto, L. y Tapia, K. (2009). Psychometric properties of the quality of life questionnaire KIDSCREEEN-27 in Chilean adolescents. Terapia Psicológica, 27(1), 83-92.

Wiles, N. J., Northstone, K. y Lewis, G. (2009). Junk food diet and childhood behavioural problems: Results from the ALSPAC Cohort. European Journal of Clinical Nutrition, 63(4), 491-498. 